\title{
The use of surface electromyography in rehabilitating rheumatic patients after knee arthroplasty (pilot study)
}

\author{
Agnieszka Prusinowska, Arkadiusz Komorowski, Piotr Syrówka
}

Rheumoorthopaedics Clinic, National Institute of Geriatrics, Rheumatology and Rehabilitation, Warsaw, Poland

\begin{abstract}
Objectives: The aim of the conducted research was to assess muscle performance in rheumatic patients qualified for knee arthroplasty before and after surgical treatment.

Material and methods: Patients with the diagnosis of rheumatoid arthritis or a degenerative joint disease qualified for surgical treatment were examined. Three groups were analysed: 1) a control group, 2) a group of patients qualified for knee arthroplasty (G1), 3) a group of patients with one knee joint endoprosthesis qualified for the second surgery (G2). The study was carried out through a portable surface electromyography system from Noraxon U.S.A. INC., Clinical DTS and using surface electrodes. The surface electromyography (SEMG) examination was conducted twice: before and on the 10th day after the surgery. The study concerned the quadriceps femoris muscle, i.e. its straight and medial head in both lower limbs during isometric tension and active movement.

Results: The comparison of the examined muscles' activity in the control group revealed greatly increased activity during isometric tension than during active movement in both muscles. In the G1 group, the comparison of the average values of isometric tension of the examined muscles before the surgery showed slight differences between the healthy limb and the one qualified for the surgical treatment. After the surgery, significant asymmetry between the average values achieved by the healthy and the operated limb could be identified in both muscles. In the G2 group, muscle activity within the currently operated limb revealed only slight differences between the limbs before the surgery. After the surgery, there was an increase in muscle activity within the previously operated limb. Conclusions: Considerably higher average values of muscle activity during the isometric tension, when compared to the active movement in a sitting position, indicate the necessity of more widespread use of isometric tension in rehabilitating patients after knee arthroplasty.
\end{abstract}

Key words: physiotherapy, surface electromyography, knee joint endoprosthesis.

\section{Introduction}

The necessity of an increasingly thorough examination of the patient for the needs of planning the therapy and physical activity means that more and more additional tests are used to complement the classic examination. In contrast to the classic neurological electromyography (EMG), which tests the artificial muscle response to external electrical stimulation under static conditions, the surface EMG (other names used include the following: surface electromyography - sEMG, global electromyography - gEMG, kinesiological EMG, functional EMG) can be described as a study of the neuromuscular activity while doing physical exercise from a physiotherapeutic rehabilitation programme, monitoring work ergonomics or sports training [1].

The examination of muscle activity by means of the EMG during dynamic activities was launched in the 1940s [2]. The advantage of surface electromyography is the possibility to record the simultaneous functions of many muscles during a dynamic examination [3]. The sEMG evaluates the symmetry of the motor units' activity of the same muscle groups on both sides as well as 
the interaction among various synergistic or antagonistic muscles functioning within the exact tested limb [4].

In the SEMG, the reception point of electrical potentials covers in its range a certain area that is difficult to define, which is why we register the interferential functional record from numerous motor units of the muscle. Special attention should likewise be drawn to the phenomenon of "cross-talk" in which the neighbouring muscle groups, if they are also active, may influence the received signal [5].

\section{Material and methods}

The aim of the research carried out at the National Institute of Geriatrics, Rheumatology and Rehabilitation in Warsaw was to assess the muscle performance in rheumatic patients qualified for knee joint arthroplasty before and after surgical treatment. The research was approved by the bioethics commission.

\section{Research questions}

What is the activity of the rectus femoris muscle and the medial head of the quadriceps femoris muscle of the thigh during an isometric contraction and the active movement of flexion and extension in a sitting position in a patient with one and two knee joint endoprostheses?

What are the differences between the activity of the examined muscles in lower limbs with and without the knee joint endoprosthesis?

What are the differences in muscle activity before and after the surgery in a patient with one and two knee joint endoprostheses?

\section{Research material}

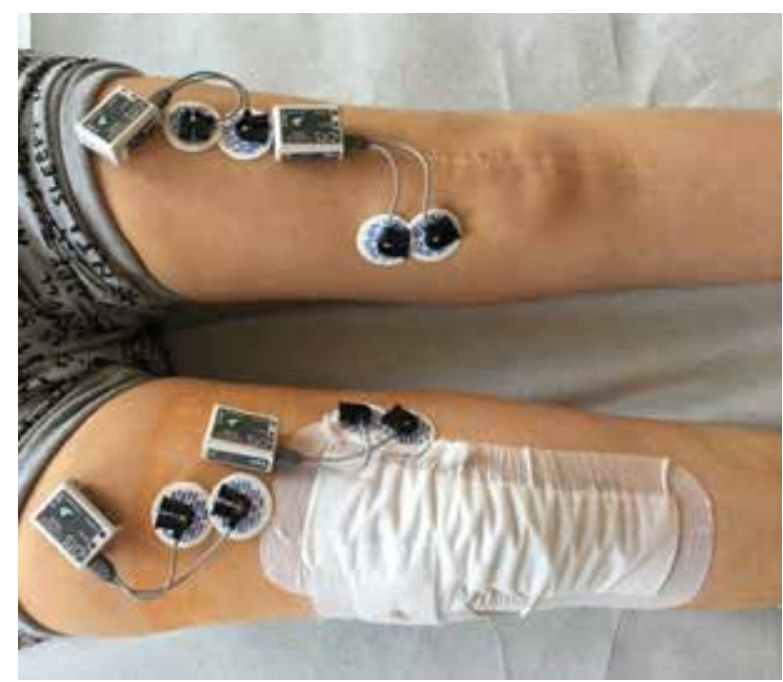

Fig. 1. Location of the registering electrodes during the sEMG test - group G2.
The criteria for including a patient in the research programme involved the diagnosis of rheumatoid arthritis or a degenerative joint disease and the qualification for the knee arthroplasty. The patient was staying at the Rheumoorthopaedics Clinic in the National Institute of Geriatrics.

The exclusion criteria included contractures in the area of knee joints that prevented 90-degree flexion and extension, destructive changes of other joints that inhibited taking up the position in which the research was conducted, i.e. lying on the back with a straight lower limb and sitting with legs down.

Risk assessment for the patient - non-invasive testing. Three groups of patients were examined. Control group $(K)$ - healthy individuals who were not diagnosed with any joint diseases or pain in the knee joints 16 patients (13 women, 3 men), average age 63.5. Test group (G1) - 10 patients qualified for knee arthroplasty - 8 women and 2 men, average age 56.4. Test group (G2) - 8 patients with one knee joint endoprosthesis qualified for the second knee joint replacement surgery -6 women and 2 men, average age 62.1.

\section{Research methodology}

The study was conducted using a portable surface electromyography system from Noraxon U.S.A. INC., Clinical DTS. Tests were carried out using surface electrodes. The surface electrodes were placed in accordance with the SENIAM (Surface ElectroMyoGraphy for the NonInvasive Assessment of Muscles) recommendations on the right and left limb and recorded signals from the following muscles: rectus femoris (RF) and vastus medialis oblique (VMO). SENIAM is a European research programme containing a number of guidelines for the selection of the type of electrodes, their location, muscle anatomy and functions, muscle group tests as well as signal processing and equipment conditions (Fig. 1) [6].

The sEMG examination of each patient was carried out twice: before the surgery and on the $10^{\text {th }}$ day after the surgery. The study concerned the musculus RF - the straight head of the quadriceps femoris muscle and musculus VMO, i.e. the medial head of the quadriceps femoris muscle.

The test consisted of two stages:

- lying on the back - isometric tension of the quadriceps femoris muscles (10 s), the so-called MVC test (maximum voluntary contraction test),

- sitting with legs down - active extension in the knee joint and flexion up to 90 degrees (3 repetitions -10 s). 
$A_{25}$
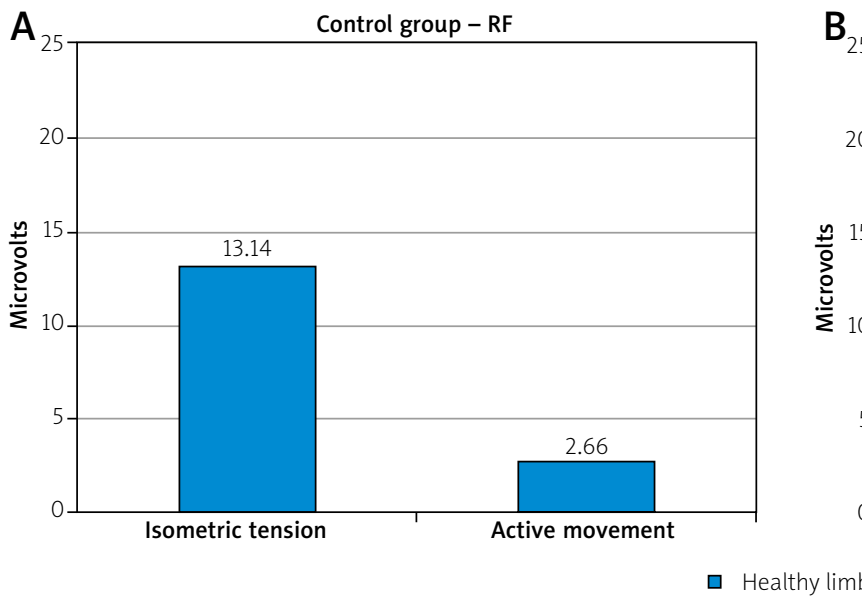

$\mathrm{B}_{25}$ Control group - VMO

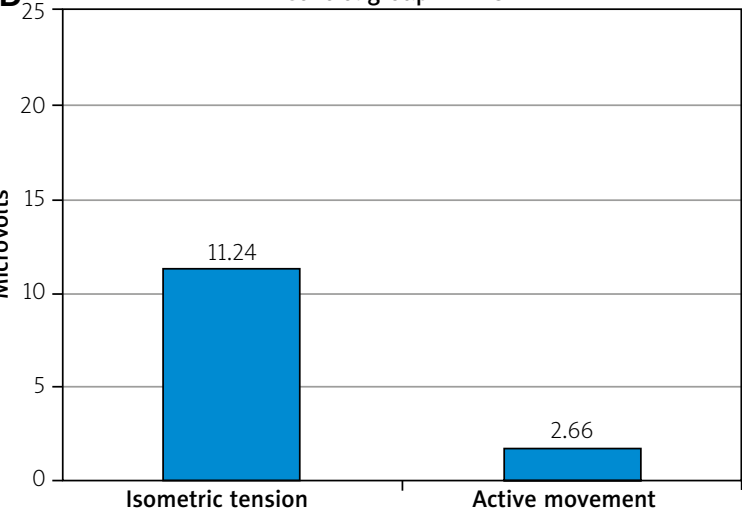

Fig. 2. Control group. Average bioelectric voltage values (microvolts) for the straight head (A) and the medial head (B) of the quadriceps femoris muscle obtained during isometric tension and active movement in the control group.
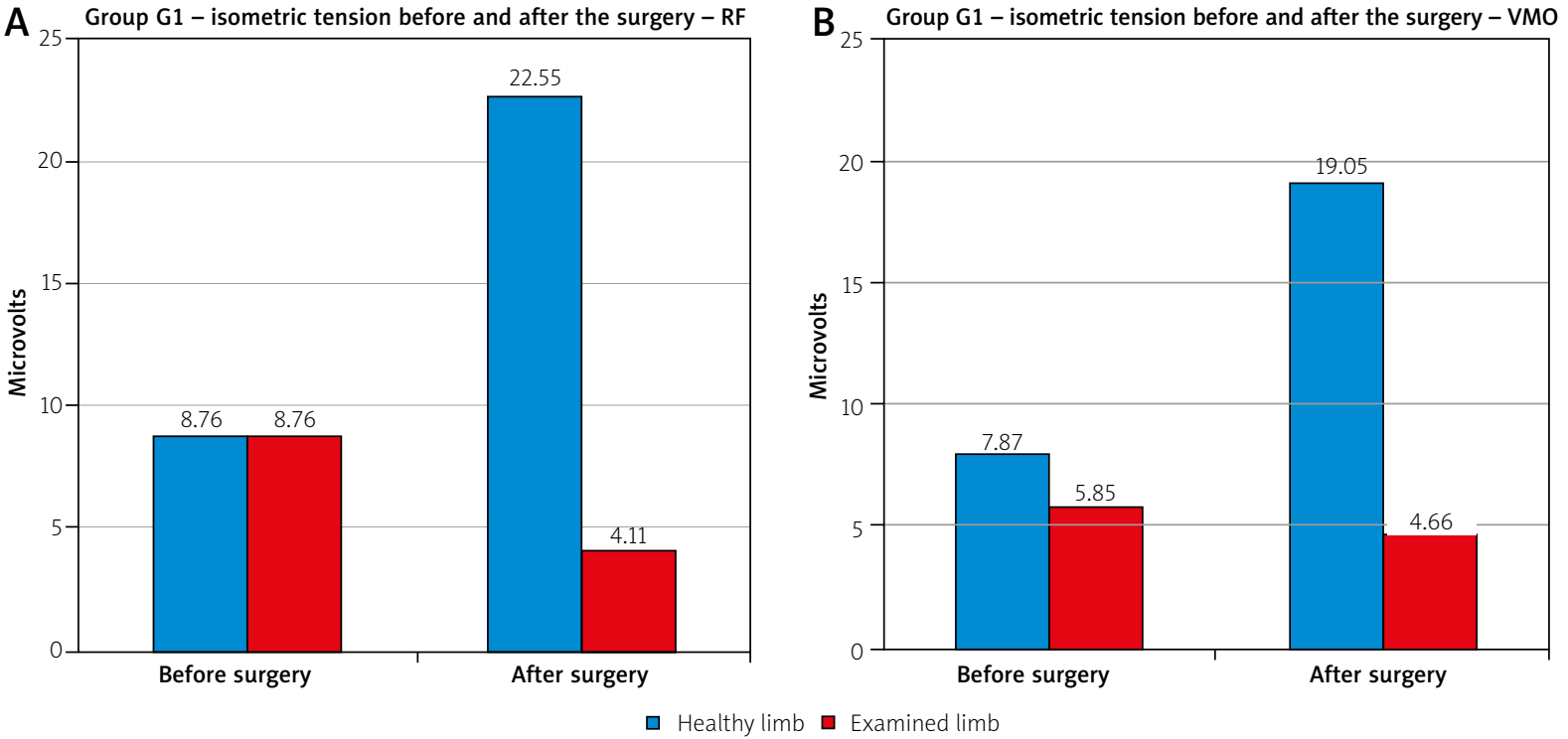

Fig. 3. Group G1. Average bioelectric voltage values (microvolts) for the straight head (A) and the medial head (B) of the quadriceps femoris muscle obtained during isometric tension before and after surgical treatment.

\section{Research findings}

The comparison of the activity of the examined muscles in the control group indicated considerably more activity during isometric tension than during active movement in a sitting position. The above applies to both the rectus femoris muscle and the medial head (Fig. 2).

In the G1 group of patients, the comparison of the average values of the isometric tension of the rectus femoris muscles of the thigh and the medial head of the quadriceps femoris muscle before the surgery reveals slight differences between the healthy limb and the one qualified for sur- gical treatment. The lowest values can be observed in the case of the medial head (VMO) of the quadriceps femoris muscle in the lower limb qualified for knee arthroplasty.

However, after the surgery, significant asymmetry between the average values achieved by the healthy and the operated limb could be identified in both examined muscles. While the activity of RF and VMO decreased in the operated limb, it increased in the healthy limb, considerably exceeding the results obtained before the surgery (Fig. 3).

In the G2 group of patients qualified for the second knee joint endoprosthesis, the activity of the RF and 
A Group G2 - isometric tension before and after the surgery - RF

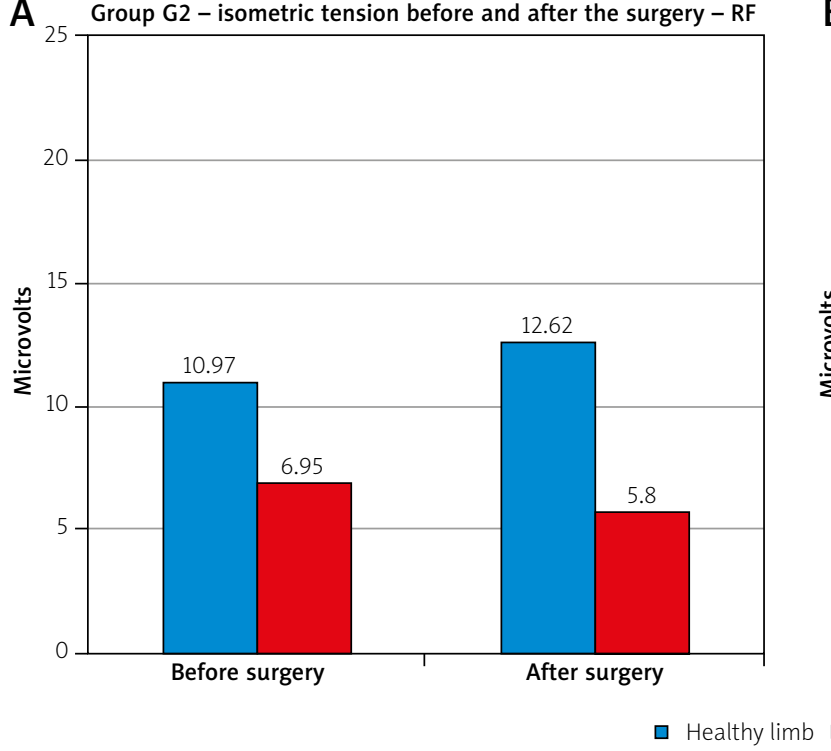

B Group G2 - isometric tension before and after the surgery - VMO

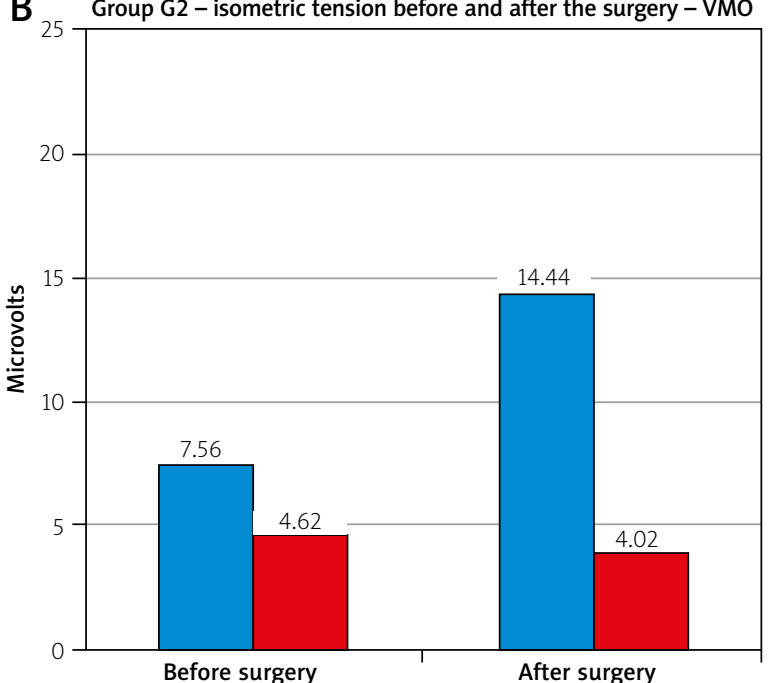

Fig. 4. Group G2. Average bioelectric voltage values (microvolts) for the straight head (A) and the medial head $(B)$ of the quadriceps femoris muscle obtained during isometric tension before and after surgical treatment.

A Group G1 - active movement before and after the surgery - RF

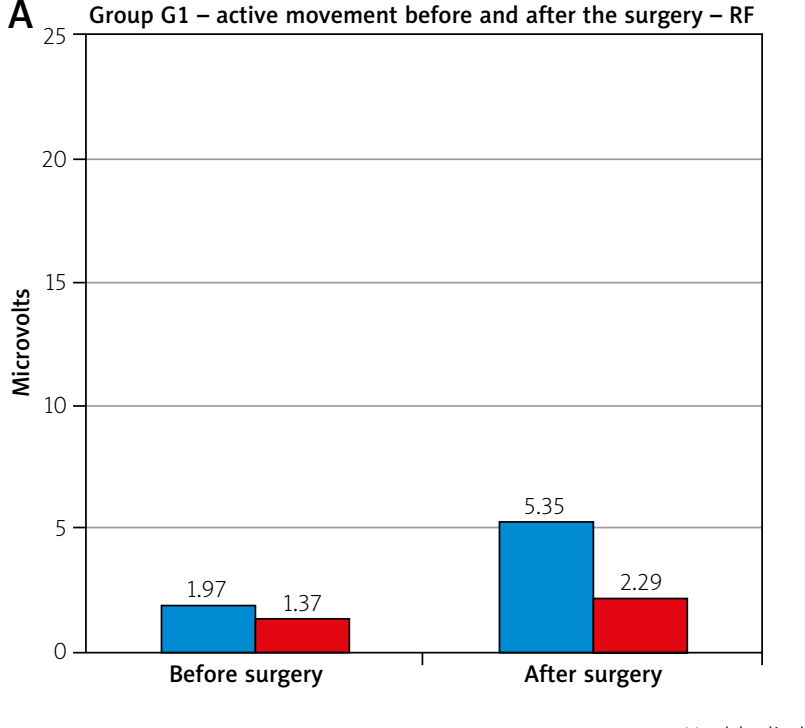

B Group G1 - active movement before and after the surgery - VMO

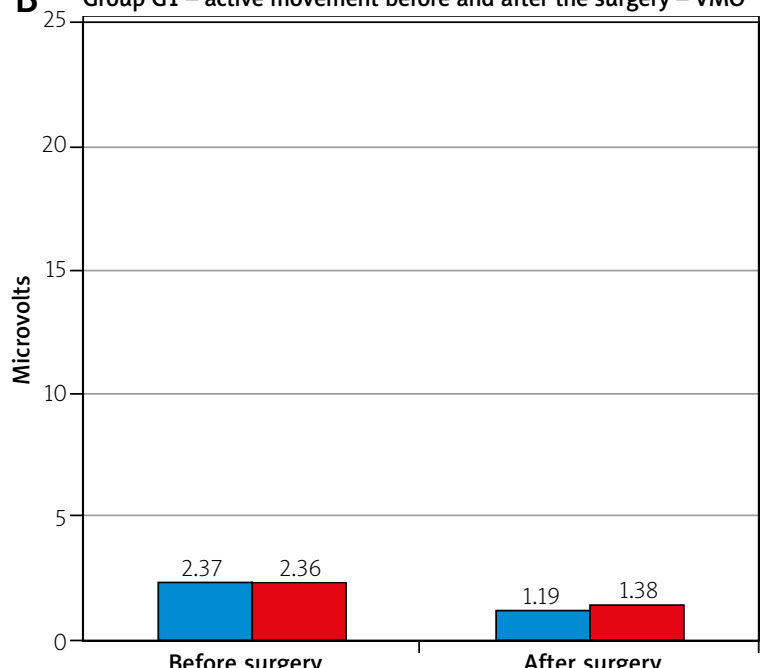

Before surgery

After surgery

Fig. 5. Group G1. Average bioelectric voltage values (microvolts) for the straight head (A) and the medial head $(B)$ of the quadriceps femoris muscle obtained during active movement before and after surgical treatment.

VMO muscles during isometric tension before the surgery revealed lower average values in the limb awaiting surgical treatment. After the surgery, there was an increase of muscle activity within the previously operated limb and a slight decrease in the limb that had just undergone the surgical treatment when compared to the condition before the surgery (Fig. 4).
Analysing the average values of the medial head activity, it can be stated that its activity before the surgery is reduced in both test groups in comparison to the control group - a slightly larger reduction in the G2 group, i.e. patients with 2 knee joint endoprostheses. However, after the surgery, an increase in the activity of the non-operated limb was observed in both groups, with 
A Group G2 - active movement before and after the surgery - RF

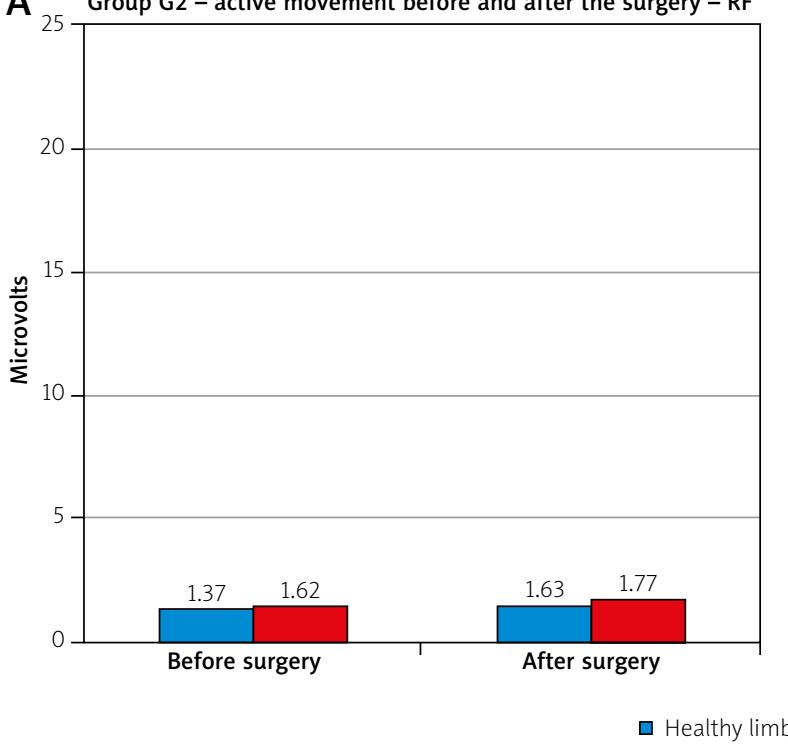

B Group G2 - active movement before and after the surgery - VMO

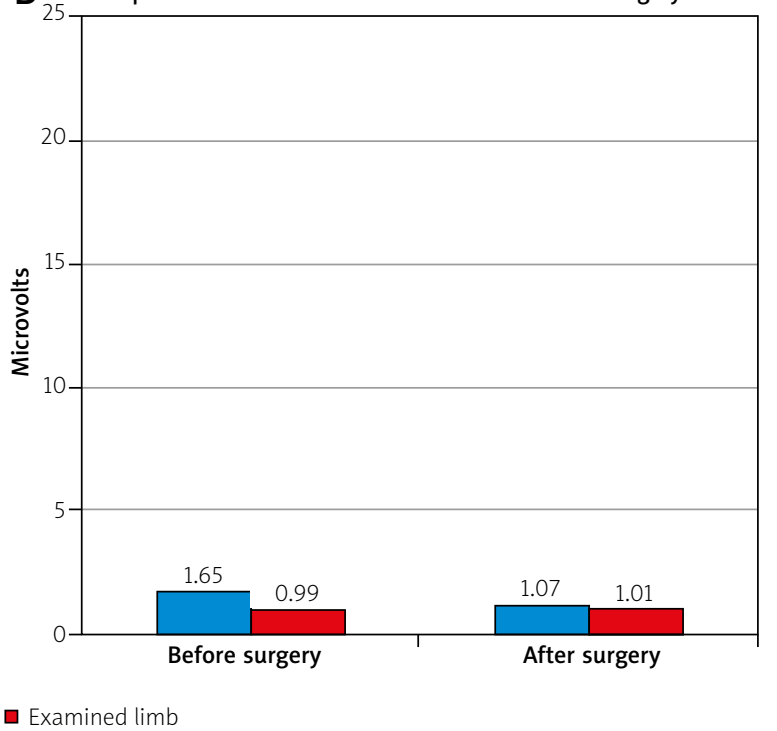

Fig. 6. Group G2. Average bioelectric voltage values (microvolts) for the straight head (A) and the medial head $(B)$ of the quadriceps femoris muscle obtained during active movement before and after surgical treatment.

values exceeding the results achieved in the control group. There was a decrease of the values within the operated limb in both groups.

In the area of the rectus femoris muscle before the knee arthroplasty, there was lower muscle activity in both test groups compared to the control group. In the G1 group, symmetry between the healthy and operated limb is visible while, in the G2 group, the activity of muscles in the non-operated limb increased. Asymmetry between the limbs appeared after the surgery in the G1 group. The values achieved by the straight head of the quadriceps femoris muscle in the healthy limb increased significantly, exceeding even the results of the control group, whereas they decreased by half in the operated limb. In the G2 group, an increase of the values in the non-operated limb (comparable to the results of the control group) can also be observed together with a slight decrease of the values in the limb after the surgery.

In the study of the RF muscle's activity during the active movement in the G1 group before the surgery, the obtained average values appear to be lower than after the surgery. After the surgical treatment, a significant increase in the activity within the healthy limb can also be seen. In the case of the VMO medial head, there were smaller differences between the limbs and, after the surgery, lower average values were observed in both limbs during the active movement (Fig. 5).

In the group of patients with 2 endoprostheses, the obtained average values of muscle activity were very low in both examined muscles before as well as after the surgery (Fig. 6).

\section{Discussion}

The assessment of muscle activity in patients diagnosed with a rheumatic disease treated surgically is an extremely important issue due to the muscular dystrophy characteristic for this group. Weak muscles are the factor which reduces their independence and determines an increased risk of falls. Muscles and their activity, during the examination of motor organs, are usually assessed only through palpation and observation. The use of surface electromyography enables the evaluation and monitoring of muscles' activity and their behaviour in real time [7].

The conducted tests checked the activity of the quadriceps femoris muscle, i.e. the straight head (RF) and medial head (VMO), in both limbs before and after the surgical treatment during isometric tension and active movement while sitting with legs down. Patients of both the G1 and G2 groups participated in the standard post-operative rehabilitation programme from the very first day after the surgery. On the $10^{\text {th }}$ day after the surgery (second examination), they walked on crutches independently, actively bent the operated knee joint up to a 90-degree angle when sitting and actively straightened it to the full extent.

Isometric muscle tension is the first exercise performed after knee arthroplasty and is considered to be 
an exercise engaging muscles the least. It was assumed that the activity of the examined muscles during the active movement in a sitting position, in which the muscle has to overcome the weight of the limb against the force of gravity, should have been greater than during the isometric tension in the supine position. The obtained results, however, demonstrate different values.

The comparison of the activity of the examined muscles in the control group revealed considerably heightened activity during isometric tension than during active movement in a sitting position. The above applied to both the medial and straight head of the quadriceps femoris muscle. In a patient after knee arthroplasty, this can be explained by pain which would be more acute during the active movement in the operated limb. Nonetheless, pain is not a differentiating factor in the control group. Hence, it could be stated that the patient tightened the examined muscles to the maximum during the isometric tension. However, during the active movement, the patient was focused on performing the movement task, i.e. extension and flexion of the knee joint, in the most economical way - not requiring the involvement of as many muscle motor units.

Significant differences in the isometric muscle tension in the test groups (G1, G2) both before and after the surgery can also be observed. The average values obtained after the surgery decreased in the operated limb. Such results can be explained by pain that patients experience after the surgical treatment as well as by the fear of activating the operated joint.

It was found that the average bioelectric voltage values in the healthy limb increased decidedly. The above may be due to the fact that patients, instinctively avoiding any activity of the operated limb, transfer the weight to the healthy limb during physical activities. Consequently, the healthy limb becomes the supporting one, ensuring stability and a sense of security.

The obtained results indicate the necessity of a more widespread use of isometric tension when rehabilitating patients, and not only after total knee replacement surgery. This seems very important in rehabilitating rheumatic patients, particularly those at an advanced age, who often limit their physical activity, which leads to muscle weakness, frequently even muscular atrophy. It is a type of activity that, if properly performed, does not excessively overwhelm the cardiovascular system, allows seniors to retain physical fitness for longer and prevents their premature exclusion from society.

The sEMG test results are influenced by various factors that should be taken into account when conducting the studies and analysing the results, e.g. a too low or too high ambient temperature. It should remain within the range of $22^{\circ} \mathrm{C}$ to $25^{\circ} \mathrm{C}$ [8]. This is the optimal tempera- ture in which there is no acceleration or slowing down of physiological processes occurring in the human body and, thus, it does not affect the muscle work in any way [9].

According to Ohm's law, tissue impedance is the main factor influencing the reading of the electrical activity of muscles. The high impedance and susceptibility to an uncontrolled change of apparent resistance, due to changes in humidity and temperature of skin layers, also have a significant impact on the result of the surface electromyography test. A different distance of surface electrodes from the muscles that are the source of potentials is likewise one of the elements affecting the system's impedance [10]. Both the postoperative swelling and the swelling which may occur in the course of joint inflammation in rheumatic diseases as well as fatty tissue of various thickness (depending on BMI) will affect the measurement results.

In recent years, what have been arousing increasingly greater interest are the methods of analysing the parameters of single motor unit action potentials (MUAP) when using surface electromyography with multi-channel reception, enabling one, inter alia, to estimate the number of active motor units, conduction velocity and muscle fibre length or to determine the zone of potential denervation of the examined muscle $[4,6]$.

Local muscle fatigue may also have an impact on the results of bioelectric signal analysis. Muscle changes occurring due to fatigue are visible in the electromyogram recording through the changed values of the EMG signal parameters [11]. Candotti et al. [12], on the basis of a study on patients with low back pain, claimed that electromyographic tests provide the opportunity to observe early changes in the muscles' functioning manifesting as fatigue, which is visible through the reduced frequency of the recording during physical activity.

Therefore, despite the fact that the advantages of electromyography are its non-invasiveness and painlessness, the undoubted disadvantages should include the limited possibility of using this test method - applied exclusively for examining muscles lying directly under the skin. It is not possible to use this method to study muscles from the deep layers whose functioning is often crucial to maintain the mobility and stability of locomotor organs. Moreover, while the reliability of this method of analysis in relation to isometric exercise has been repeatedly confirmed, less is known about its reliability in relation to dynamic exercise, especially ballistic movements [9].

Electromyography, in addition to its basic application for diagnosis of the muscle's electrical activity, has also evolved as a therapeutic method that can be applied by patients themselves, both during their stay at hospital and after returning home using the rented 
equipment. It became possible due to biofeedback training using an electromyograph. Biofeedback is a therapy which involves sending the feedback signals about changes in the physiological state of the patient's body owing to which patients can, by observing the results, consciously modify the functions that are not controlled consciously under normal conditions in this case it is muscle tension. The electromyographic analysis can provide valuable information as to the relative muscle activity required by a given exercise and help to determine the optimal body position for the exercise [7]. Thanks to the use of this method, patients can independently and properly perform exercises they are assigned, both isometric and active but slow ones.

The feedback response that patients receive during the therapy allows them to perform active but slow exercises so effectively that the work of the examined muscle is comparable to the results which can be achieved during the properly done isometric exercises.

Sjors et al. [13] suggested applying EMG for therapeutic purposes as a kind of specific muscular biofeedback due to which patients would learn how to relax muscles. They used surface electromyography for the assessment of people suffering from pain to evaluate the strength and endurance of muscles. They presented the results of the research conducted on a group of women with myalgia occurring in the trapezius muscle. Indeed, EMG biofeedback has become a method helpful in the work of physiotherapists and extremely advantageous in teaching how to do the exercise correctly. The electromyographic record can be essential in learning to properly tighten the muscles and in the right order [14].

The simultaneous observation of the results when performing the strengthening exercise may be a stimulus for patients to do it more thoroughly, giving them at the same time a sense of responsibility for the effect of their own medical treatment. The EMG biofeedback can also serve as a tool enhancing the adoption of the correct posture. It can help people having a sedentary job at the computer to eliminate inappropriate habits. Placing the electrodes in the neck and shoulder area and observing the recording on the monitor may make the patient aware of the disproportionate muscle involvement when working, e.g. the elevation of the shoulders or excessive activity of the descending part of the trapezius muscle during typing [14].

According to various authors, surface electromyography may be helpful in assessing the condition of patients suffering from pain. The observed changes in the recording may include a decrease of the electromyogram's amplitude value in effort conditions and an increase of the amplitude value in resting conditions as well as a decrease of the frequency value in effort con- ditions. Inter alia, Roy and Oddsson [15] consider that surface electromyography can be useful in assessing the inhibition of muscles occurring secondarily in relation to pain and in evaluating the activity associated with low back pain

On the basis of the research on patients with low back pain, Candotti et al. [12] conclude that electromyographic tests enable one to observe early changes in the muscles' functioning manifesting as fatigue, which is visible through the reduced frequency of the recording during physical activity.

Pullman et al. [16] also refer to research in which the possibilities of using electromyography to assess muscle fatigue are presented. The increase of this phenomenon may be indicated by a decrease in the frequency of the recording when performing physical exercise.

Sjors et al. [13] suggest using surface electromyography for the assessment of people with pain discomforts. The sEMG could be applied to evaluate the muscle strength and endurance, which they investigated in studies conducted on a group of women with myalgia in the trapezius muscle. They observed increased muscle tension in women with trapezius muscle pain and advised that the EMG examination could also be used for therapy as muscle biofeedback due to which patients would learn how to relax muscles [14].

Electromyographic tests, which are a source of objective information about the patient's condition, seem to have widespread application in physiotherapy, primarily as a tool complementing physiotherapeutic activities. In the era of increasing use of technology and evidence-based medicine, the use of electromyographic tests should become common due to their non-invasiveness and painlessness, simplicity to carry out the examination after having received proper training and the opportunity to objectively document the rehabilitated patient's condition [17].

The conducted pilot study, whose aim was to evaluate the possibility of using surface EMG in examining rheumatic patients after surgical treatment, has yielded interesting results that can be used to modify the rehabilitation programme not only for postoperative patients. Therefore, we plan to continue and expand the already started research so that the obtained results can initiate a faster return of the treated patients to complete physical activity.

\section{Conclusions}

Significantly higher average values of muscle activity during isometric tension, when compared to active movement in a sitting position, indicate the necessity of 
more widespread use of isometric tension in rehabilitating patients after knee arthroplasty.

The authors declare no conflict of interest.

\section{References}

1. Konrad P. ABC of EMG - A Practical Introduction to Kinesiological Electromyography, Noraxon Inc. USA, Version 1.0 April 2005.

2. Piotrowska SE, Majchrzycki M. Surface Electromyography A Review. Issue Rehabil Orthop Neurophysiol Sport Promot 2014; 6: 19-28.

3. Karthick, PA, Ghosh DM, Swaminathan R. Surface Electromyography Based Muscle Fatigue Detection Using High-Resolution Time-Frequency Methods and Machine Learning Algorithms. Computer Methods and Programs in Biomedicine 2018; 154 : 45-56.

4. Witkowska A. Importance of the Global Electromyography in Neurophysiological Diagnostics, a Concept of Single Motor Units Action Potentials Analysis (doctoral dissertation) Poznan University of Medical Sciences, 2010.

5. Sobota G. Electromyography and Its Application in Disorders of the Chewing Apparatus. Dysfunctions of the Chewing Organ. Your Dental Review 2012; 6: 57-61.

6. Staudenmann D, Roeleveld K, Stegeman DF, et al. Methodological Aspects of sEMG Recordings for Force Estimation - A Tutorial and Review. J Electromyogr Kinesiol 2009; 20: 375-387.

7. Türker H, Sözen H, Surface Electromyography in Sports and Exercise. In: Electrodiagnosis in New Frontiers of Clinical Research, Turker H (ed.). InTech, Rijeka 2013.

8. Goch M. Function of Selected Muscles and Conductivity of Motor Fibers after Total Hip Alloplasty with a Lateral Approach (doctoral dissertation). Poznan University of Medical Sciences, Faculty of Medicine II, 2014.
9. Fauth M, Petushek EJ, Feldmann CR, et al. Reliability of Surface Electromyography during Maximal Voluntary Isometric Contractions, Jump Landings, and Cutting. I Strength Cond Res 2010; 24: 1131-1137.

10. Woźniak K, Lipski M, Lichota D, et al. Surface Electromyography in Dentistry: EMG 8 System - Bluetooth. Implantoprot 2008; 3: 52-55.

11.Vigotsky AD, Halperin I, Lehman GJ, et al. Interpreting Signal Amplitudes in Surface Electromyography Studies in Sport and Rehabilitation Sciences. Front Physiol 2018; 8: 985.

12. Candotti CT, Loss JK, Pressi AM, et al. Electromyography for assessment of pain in low back muscles. Phys Ther 2008; 88: 1061-1067.

13. Sjörs A, Larsson B, Dahlman J, et al. Physiological Responses to Low-Force Work and Psychosocial Stress in Woman with Chronic Trapezius Myalgia. BMC Musculoskelet Disord 2009; 10: 63.

14. Wytrążek M, Huber J, Zagłoba-Kaszuba A, et al. Possibilities of Using Electromyographic Studies in the Physiotherapeutic Practice. In: Directions in the Development of Clinical Neurophysiology, Physiotherapy and Manual Therapy, Hubera J, Wytrążek M, Kabscha A (eds.). Poznan University of Medical Sciences, 2010

15. Roy SH, Oddsson LI. Classification of Paraspinal Muscle Impairments by Surface Electromyography. Phys Ther 1998; 78: 838-851.

16. Pullman SL, Goodin DS, Marquinez Al, et al. Clinical Utility of Surface EMG: Report of the Therapeutics and Technology Assessment Subcommittee of the American Academy of Neurology. Neurology 2000; 55: 171-177.

17. Kondrat A, Huber J, Ziółkowska A, et al. Evaluation of Mckenzie Treatment with Surface Electromyography in Patients with Lumbo-Sacral Discopathy - Pilot Studies. Issue Rehabil Orthop Neurophysiol Sport Promot 2014; 8: 26-42. 